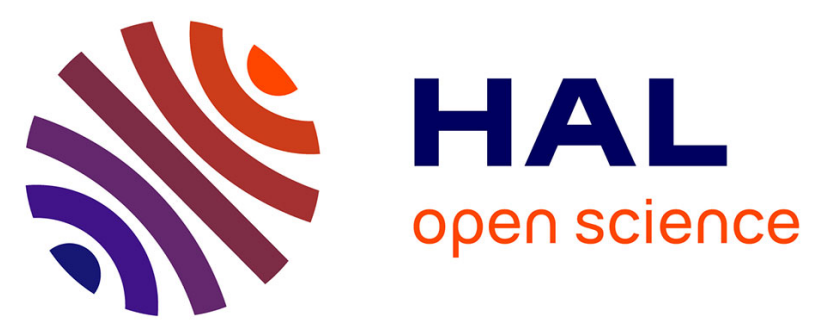

\title{
Simulation and Experiments on Magnetic MicroForces for Magnetic MicroRobots Applications.
}

Lefeng Wang, Mohamed Dkhil, Aude Bolopion, Patrick Rougeot, Stéphane Régnier, Michaël Gauthier

\section{- To cite this version:}

Lefeng Wang, Mohamed Dkhil, Aude Bolopion, Patrick Rougeot, Stéphane Régnier, et al.. Simulation and Experiments on Magnetic MicroForces for Magnetic MicroRobots Applications.. The Third International Conference on Manipulation, Manufacturing and Measurement on the Nanoscale, 3M-NANO 2013., Jan 2013, China. pp.1-6. hal-00873409

\section{HAL Id: hal-00873409 \\ https://hal.science/hal-00873409}

Submitted on 15 Oct 2013

HAL is a multi-disciplinary open access archive for the deposit and dissemination of scientific research documents, whether they are published or not. The documents may come from teaching and research institutions in France or abroad, or from public or private research centers.
L'archive ouverte pluridisciplinaire HAL, est destinée au dépôt et à la diffusion de documents scientifiques de niveau recherche, publiés ou non, émanant des établissements d'enseignement et de recherche français ou étrangers, des laboratoires publics ou privés. 


\title{
Simulation and Experiments on Magnetic MicroForces for Magnetic MicroRobots Applications
}

\author{
Lefeng Wang ${ }^{1}$, Mohamed Dkhil ${ }^{2,3}$, Aude Bolopion ${ }^{2}$, Patrick Rougeot ${ }^{2}$, Stéphane Régnier ${ }^{3}$ and Michaël Gauthier ${ }^{2}$ \\ ${ }^{1}$ State Key Laboratory of Robotics and System, Harbin Institute of Technology, Harbin, 150080, China \\ ${ }^{2}$ FEMTO-ST Institute, AS2M department, Université de Franche-Comté/CNRS/ENSMM/UTBM, 24 rue Savary, 25000 Besançon, \\ France \\ ${ }^{3}$ Institut des Système Intelligents et de Robotique (ISIR), Université Pierre et Marie Curie/CNRS UMR 7222, 75005 Paris, France \\ lefengwang@hit.edu.cn, mohamed.dkhil@femto-st.fr, regnier@isir.upmc.fr
}

\begin{abstract}
Magnetic microrobots have a wide variety of applications in micro/nano-manipulation, micro/nano sensing and biomedical fields. Untethered magnetic microrobots are usually driven by a group of electromagnetic coils. To control the magnetic microrobots trajectory, it is of utmost importance to know the magnitude of the magnetic force applied on them. In this paper finite element simulations are proposed to derive the magnetic field produced by an iron core coil. A three dimensional static magnetic analysis is also performed to simulate the magnetic force applied on a magnetic microrobot. The simulation results are validated by experimental measurements. A teslameter is used to measure the magnetic field on the central axis of the coil. A magnetic microrobot (less than $400 \times 400 \mu \mathrm{m}^{2}$ ) is fabricated and glued to a force sensor to measure the magnetic force applied on it. The measurement results are in good agreement with the simulation and show the effectiveness of the magnetic simulation.
\end{abstract}

Keywords-magnetic microrobot; magnetic field measurement; magnetic microforce

\section{INTRODUCTION}

Untethered magnetic microrobots have a wide number of potential applications in scientific and technical areas. They can be utilized to manipulate or assemble micro/nano-parts to build complex and functional micro/nano-mechanical systems. They can also be used to sense at micro/nano-scale under environments with extremely limited spaces. In biomedical fields, they can perform single cell manipulation, minima invasive microsurgery with specialized tools or even be put into the human body to execute drug delivery tasks [1]-[3].

So that the above mentioned applications prove to be realistic and reliable, a precise driving and control of the magnetic microrobots must be performed. In general, there are two ways to generate controlled magnetic fields: mobile permanent magnets [4] and fixed electromagnets. The control of the trajectory of the magnetic microrobot proves to be more flexible using electromagnets since its position is set by controlling the current applied to the coils. This technique has thus received a lot of attentions. In recent years, several magnetic microrobots and corresponding driving setups have been developed [5]. Mag- $\mu$ Bot was developed in Carnegie Mellon University, and it is driven by three pairs of coils [6].
An electromagnetic system named OctoMag was demonstrated in ETH Zurich, and it consists of four pairs of coils [7]. A paramagnetic microparticle system was developed in University of Twente [8]. In ISIR and FEMTO-ST Institute, a magnetic microrobot called 'Magpier' has also been proposed [9].

For driving electromagnet setups of microrobots, air-core electromagnets can be utilized, but the magnetic fields are not sufficient in many cases to produce large forces to perform microtasks unless big-volume coils are used. The core-filled coil is a good choice to increase the magnetic field gradient. Unfortunately, the filled cores produce highly non-linear magnetic fields [10], and there is no analytical expression of the magnetic field that they produce even along the central axis of the coil. Finite element method (FEM) are thus necessary to determine the magnetic field.

For the microrobots themselves, they are usually fabricated using soft-magnetic materials, such as nickel. The fabrication processes are rather simple and the magnetization of ferromagnetic materials is high enough to ensure that they will experience high magnetic forces. To realize a precise control of the magnetic microrobot, it is of utmost importance to characterize the magnetic field in the close vicinity of the microrobot and to know the magnetic force applied on the robot [11]. However, due to the shape anisotropy, calculating its magnetization is difficult for geometries more complex than ellipsoids [12].

This work aims at simulating the magnetic field and the magnetic force produced by an electromagnet coil with an iron core. The simulation will be confirmed by experimental measurements. The developed simulator will enable to design the magnetic actuation setup, to determine the workspace that can be reached by the robot and to control the locomotion of the magnetic robot precisely.

This work is organized as follows. Section II briefly presents a typical magnetic actuation system. Section III performs simulations and measurements of the magnetic field produced by a coil with an iron core. Section IV illustrates the simulation and measurement of the magnetic force applied to a microrobot along the central axis of the coil. This paper is concluded in Section V. 


\section{MAGPIER MAGNETIC MicRoRoBot PlatForM}

Most of the non-contact magnetic actuation platforms present similar architectures. They are composed of coils used to control the position of a ferromagnetic or diamagnetic particle by setting the current on each coil [3], [6], [7], [9]. The analysis proposed in this paper will be illustrated on the Magpier magnetic driving platform (Erreur! Source du renvoi introuvable.). It consists in four coil assemblies, a base and four connecting parts between them. The four coil assemblies are arranged orthogonally and pointing at a common center. The relative distance between them can be adjusted by moving the position of each coil assembly. A microfabricated microrobot can be manipulated in the workspace situated in the area between the coils assemblies, and they can be controlled by applying a given current on the four coils.

Since the four coil assemblies of the microrobotic platform are uniform, only one of them is considered in the following magnetic field simulation. The structure of each coil assembly is shown in Erreur! Source du renvoi introuvable. The coil assembly have an iron core in the center, to strengthen the magnetic field of the electromagnet. A plastic part is used to restrict the position of the coil.

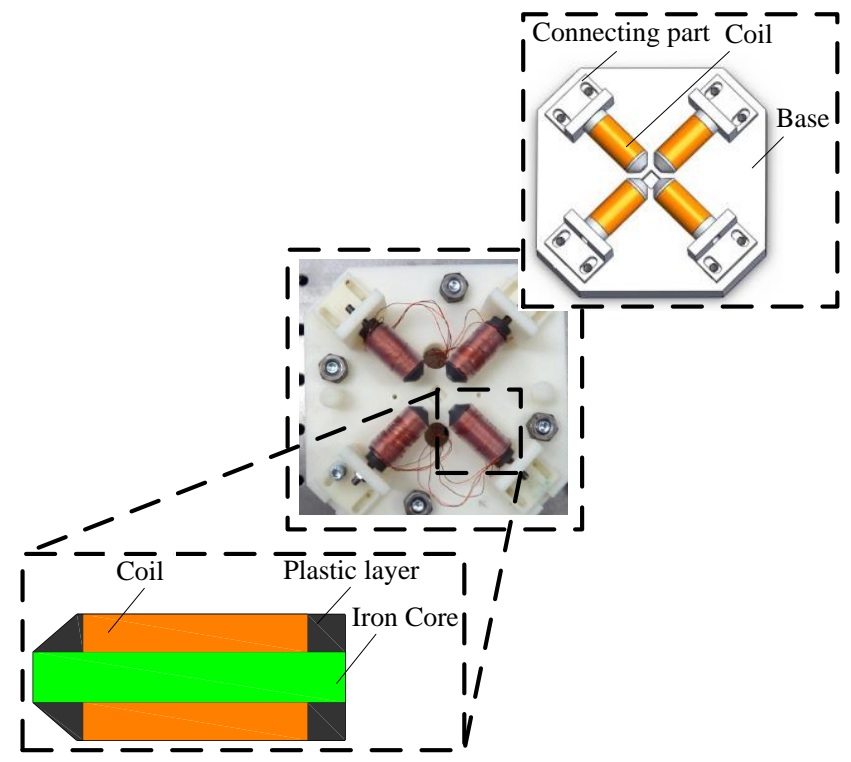

Fig. 1 Magpier magnetic microrobot platform setup

\section{SimUlation AND MEASUREMENT OF THE MAGNETIC FIELD}

\section{A. Magnetic Field Simulation}

A finite element software (Ansys software) is used to simulate the magnetic field produced by one coil of the setup. Considering that the electromagnet structure is axisymmetric along the axis of the iron core, a 2-D axisymmetric simulation of magnetic field is performed.

In the simulation, the PLANE 53 element is utilized for all the physical regions. The plastic layer is ignored in the model since it is not magnetizable. The smart meshing method is adopted, and the meshing refinement is used to improve the analysis accuracy. The numerical values of the parameters used in the simulation are shown in Table I. The built geometric model is shown in Erreur ! Source du renvoi introuvable.. A current density $J=N \times I / A_{\mathrm{c}}$ is applied on the coil, where $A_{\mathrm{c}}=L_{1} \times T$ is the cross area of the coil. The magnetic flux parallel boundary condition is applied on the exterior of the geometric model.

Table I Parameters in the simulation

\begin{tabular}{c|c}
\hline Parameter & Value \\
\hline Length of the coil $L_{1}$ & $18 \mathrm{~mm}$ \\
\hline Thickness of the coil $T$ & $3 \mathrm{~mm}$ \\
\hline Turn of the coil $N$ & 1200 \\
\hline Current of the coil $I$ & $0.4 \mathrm{~A}$ \\
\hline Length of the core $L_{2}$ & $25 \mathrm{~mm}$ \\
\hline Diameter of the core $D$ & $4 \mathrm{~mm}$ \\
\hline Permeability of the core $\mu$ & $4000 \mathrm{H} / \mathrm{m}$ \\
\hline
\end{tabular}

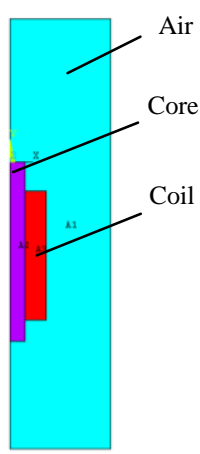

Fig. 2 The built geometric model

The 2D magnetic flux contour lines of the electromagnet are shown in Erreur! Source du renvoi introuvable. Correspondingly, the 2D axisymmetric expansion of the magnetic flux density of nodal solutions is shown in Fig. 4. From the figure, there exists maximum magnetic field intensity in the middle position of the iron core corresponding to the coil position.

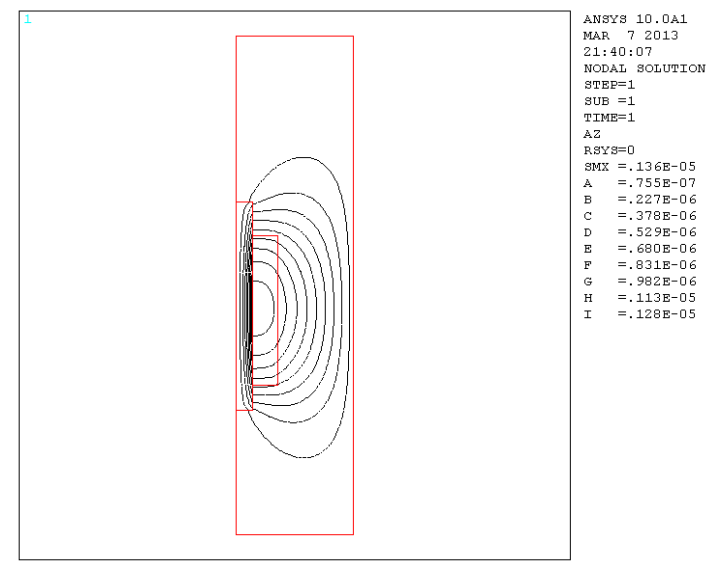

Fig. 3 2D magnetic flux contour lines

Fig. 5 shows the influence of the input currents in the coil on the magnetic fields along the central axis of the coil assembly. It indicates that the magnetic field along the central axis is proportionate to the input current at the same position. 
At the same applied current, the magnetic field becomes weak gradually with distance from the coil assembly increases. The figure gives us a basic guide to choose the work position of the microrobot, thus we can control the locomotion of the microrobot by controlling the coil current effectively.

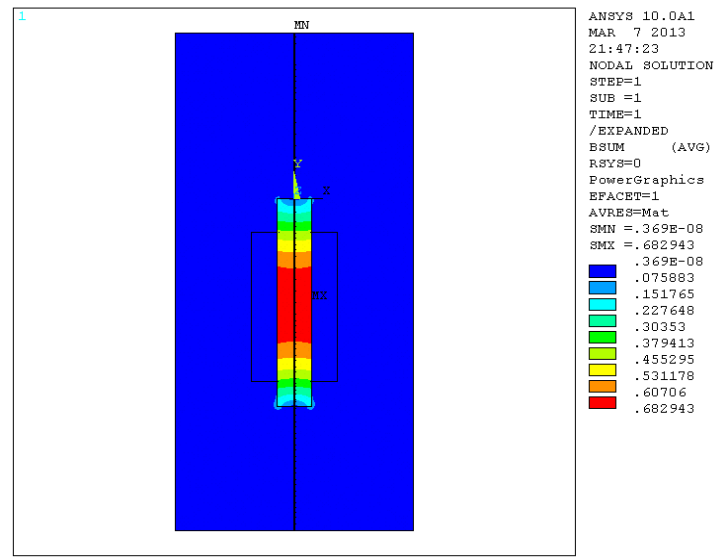

Fig. 4 2D axisymmetric expansion of the magnetic flux density

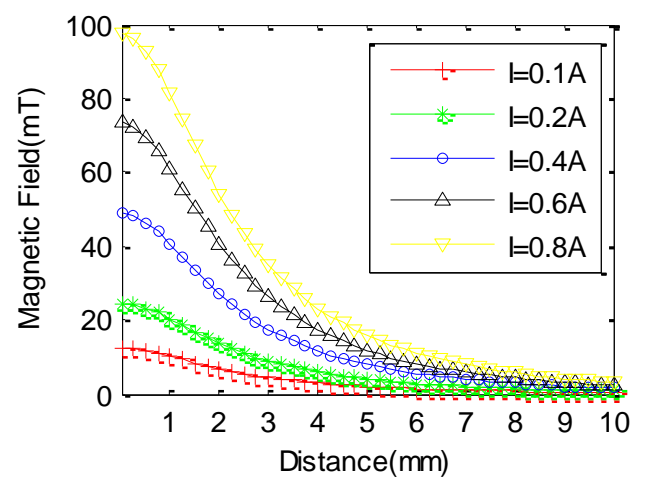

Fig. 5 Influences of the currents on the magnetic fields

\section{B. Measurement Experiments}

To validate the simulation results, experimental measurements are carried out. The magnetic field along the axis of the coil with an iron core is measured with a Gauss/Tesla meter (Model 7010 from SYPRIS Test \& Measurement Company, US). It can measure a maximum magnetic field of $3 \mathrm{~T}$ with a resolution of $0.01 \mu \mathrm{T}$. The measurement setup is shown in Fig. 6. The magnetic driving setup is fixed on a vibration-isolated platform.

To measure the magnetic field produced by an electromagnet the other three ones are removed to avoid any coupling effects. A constant current of $0.4 \mathrm{~A}$ is applied to the coil through a voltage generator and a power-amplification circuit. This is a typical value for that setup [5]. The signal generator provides a positive square wave signal of $500 \mathrm{mV}$ amplitude and $1 \mathrm{~Hz}$ frequency. A two-dimensional micropositioning stage is used to adjust the relative position of the measurement probe and the coil assembly. It is also fixed on the vibration-isolated platform.
The measurement results of magnetic field along the central axis are compared to the simulation ones in Fig. 7. The trend of the simulated result plot is similar to the experimental one. However, the simulated magnetic field is a little larger than the measured value when the distance is less than about $4 \mathrm{~mm}$. One potential factor is the difficulty of keeping the measurement probe precisely on the central axis of the coil assembly during the whole experiment process.

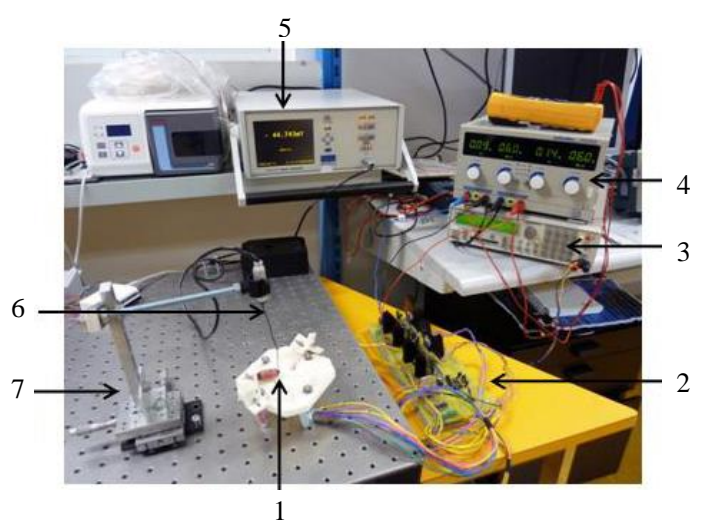

Fig. 6 Magnetic field measurement setup: 1-Coil, 2Amplification circuit, 3-Signal generator, 4-Voltage generator, 5-Measurement instrument, 6-Measurement probe, 7-Micropositioning stage

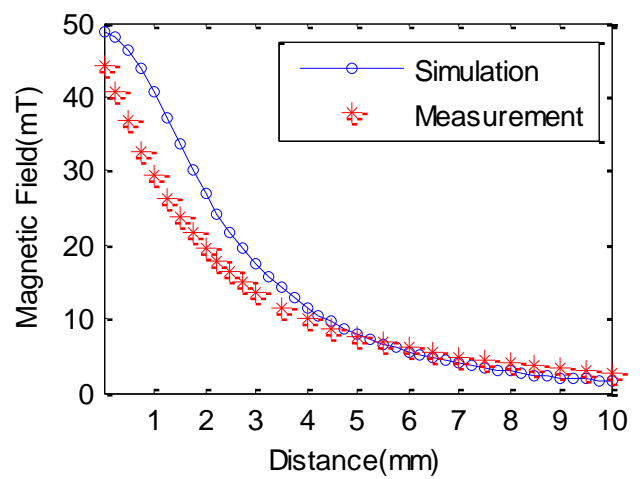

Fig. 7 Magnetic field produced by a coil on its central axis. Comparison between the simulated results and the experimental measurements

\section{Simulation AND Measurement of the MagnetiC FORCE}

\section{A. Magnetic Force Simulation}

To evaluate the force applied on a magnetic microrobot a square ferromagnetic particle is considered. When this particle is added on the system, the structure is not axisymmetric, and a three dimensional simulation is necessary to characterize the magnetic force along the central axis of the coil assembly. Fig. 8 shows the position relationship of the coil and the particle. To the noted, the dimensions are not scaled to clearly show the key structures in the figure. 
Considering the symmetric structure of the objects, $1 / 4$ model is built in the simulation. A 'racetrack' current source is defined in the suitable location, and the applied current is $0.6 \mathrm{~A}$ in the simulation. Solid 96 element is utilized, and smart element sizing was specified for meshing. Magnetic relative permeabilities of nickel $\mu_{\mathrm{r}}=1000$ was specified in the simulation. Fig. 9 shows the meshing of the simulation system including coil assembly, particle and air. Difference scalar potential (DSP) method was used to get the static magnetic solution.

The results obtained will be presented in the next section, and compared with experimental results.

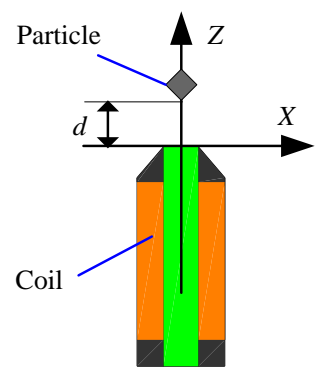

Fig. 8 Relative position of the coil and the particle

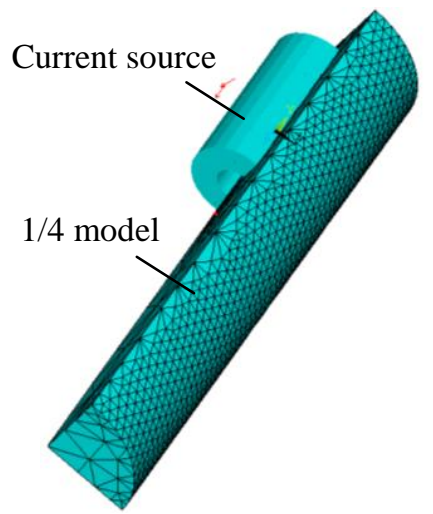

Fig. 9 Mesh and the current source used for the simulation of the magnetic force

\section{B. Magnetic Force Measurement}

A nickel particle with a square shape is firstly fabricated using micro-fabrication technologies. Nickel electrodeposition is performed on a silicon wafer. The thickness of the nickel layer is around $20 \mu \mathrm{m}$. To ensure the electrical conductivity for the electrodeposition process a thin layer (hundreds of nanometers) of chrome-copper is sputtered on the wafer. Saw dicing is used to obtain particles with the desired dimensions. The complete flow chart is given in Fig. 10.

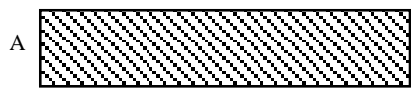

B
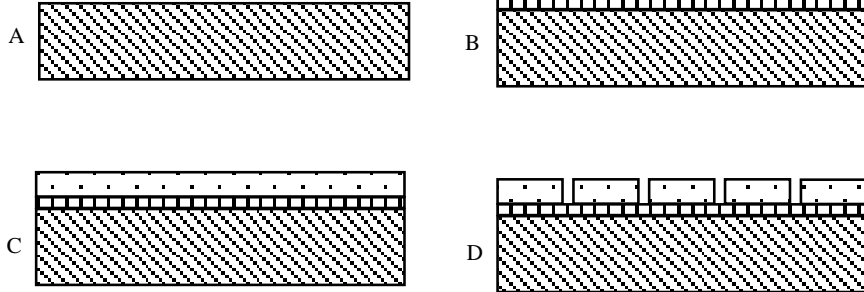

D

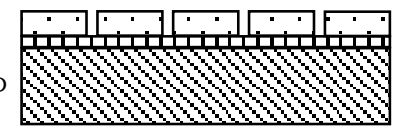

Fig. 10 Flow chart of the fabrication of the magnetic microrobot. A: the 3" silicon wafer, B: a chrome/cupper layer is sputtered on the wafer, C: a nickel layer is electrodeposited, D: microparts are obtained by saw dicing.

The measurement principle and the corresponding setup are shown in Fig. 11 a) and b) respectively. It mainly includes a microforce sensor, a three-dimension precise motion stage, a three-dimension manual stage to adjust the focus distance of the microscope, two microscopes and two CCD cameras for imaging the microparticle and the coil. A commercial microforce sensor (FT-S540 from Femto Tools Company) is adopted to measure the magnetic force along the central axis. The force range of the sensor is $180 \mu \mathrm{N}$, and the sensitivity is $90 \mu \mathrm{N} / \mathrm{V}$. It can achieve a resolution of $0.3 \mu \mathrm{N}$ at $1000 \mathrm{~Hz}$.

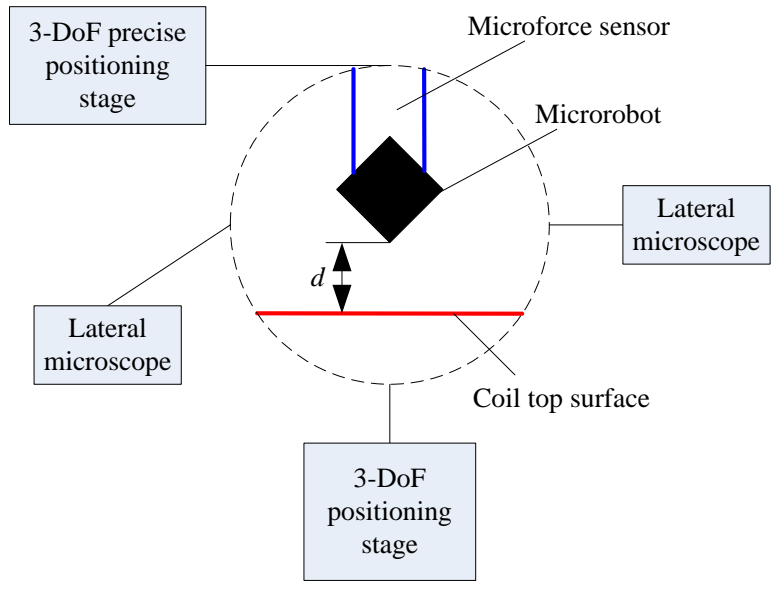

a) Measurement principle

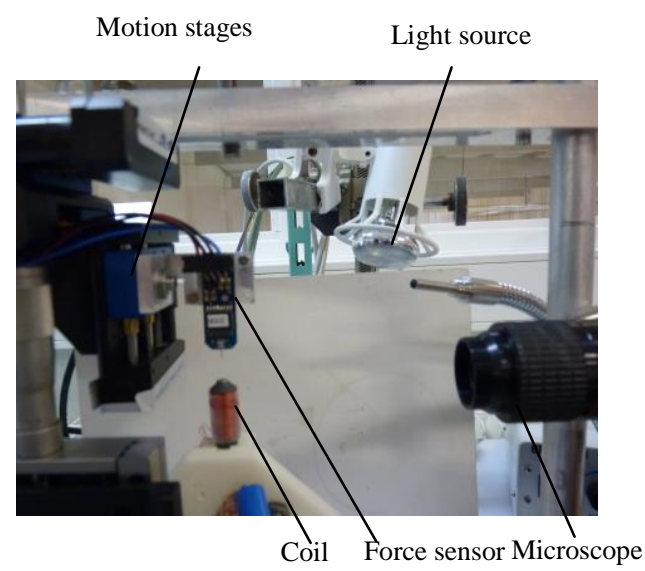




\section{b) Measurement setup}

Fig. 11 Experimental setup for measuring magnetic forces

The length of each side of the particle is around $368 \mu \mathrm{m}$ and the thickness is around $20 \mu \mathrm{m}$ according to the measurement of the optical microscope. To measure the magnetic force applied on this microrobot, it is glued on the tip of the microforce sensor with UV glue.

The force sensor is mounted on a micropositioning stage. The distance between the coil and the particle is controlled through this positioning unit. To avoid any contact between the particle and the coil, a small gap is kept between them (Fig. 12). This gap has been measured by moving the force sensor down until it contacts the coil. The calibration results show that the nearest distance between the sensor and the coil is around $59 \mu \mathrm{m}$ (Fig. 12).

The current applied through the electromagnetic coil is set to $0.6 \mathrm{~A}$. The measured magnetic force along the central axis of the coil is shown in Fig. 13. This figure presents the comparison between the simulation results and the experimental ones. When the distance between the particle and the coil is less than about $2 \mathrm{~mm}$, the measured force is a little smaller than the simulated one. On the one hand, this is probably due to inaccurate permeability assumption of the iron core in the simulation; on the other hand, the measurement environment such as local temperature due to coil heat which was not controlled strictly in our experiments could influence the measurement accuracy of microforces.

The trend of the simulated force curve is in good agreement with the experimental one. The magnetic force increases firstly and then decreases gradually. It can be attributed to the magnetic field gradient near the coil assembly. When the particle is extremely close to the coil, the magnetic field lines are almost parallel to the end surface of the coil assembly. Thus the gradient of the magnetic field could increase a little when the particle moves far from the end surface. While the nickel particle is further away from the coil assembly, the gradient of the magnetic field decreases because the magnitude of the magnetic field itself decreases gradually.

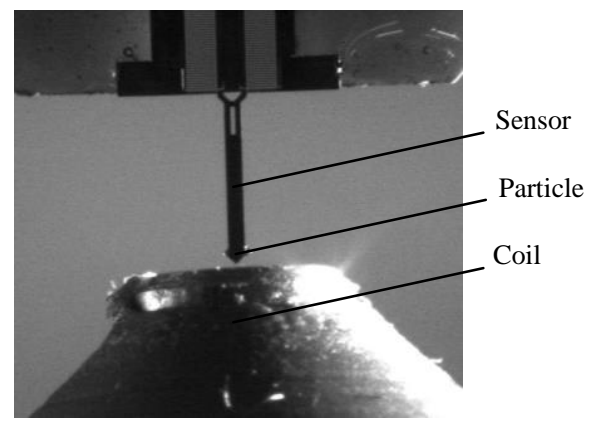

Fig. 12 Initial relative position of particle and the coil assembly in the force measurement process

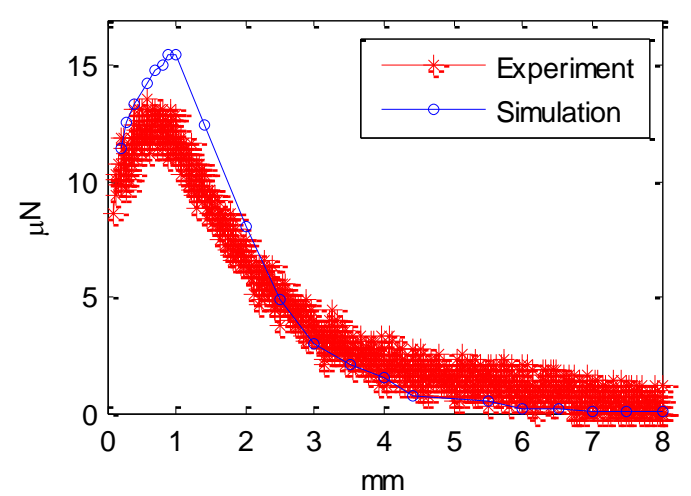

Fig. 13 Measured and simulated magnetic force

To analyze the repeatability of the force measurement, this experiments have been performed four times using the same input current in the coil $(0.6 \mathrm{~A})$. The results are provided in Fig. 14. For each trial, the maximum value of the forces as well as the corresponding distance between the coil assembly and the particle has been indicated in Table II. These results show that the force measurement has a good repeatability (the standard deviation of the maximum force is less than $1 \mu \mathrm{N}$ ). The trend of the force measurement curves is similar for the first $4 \mathrm{~mm}$. When the distance increases the system becomes more sensitive to external noises. The maximum value of the magnetic forces is around $12.63 \mu \mathrm{N}$, for a distance between the coil and the particle of around $736.9 \mu \mathrm{m}$. This proves that the magnetic actuation can apply a significant force to the microrobot to overcome the adhesion forces and the inertia force. However it is rapidly decreasing and the workspace should not be placed at a distance larger than $4 \mathrm{~mm}$ from the coil to maximize the magnetic force.

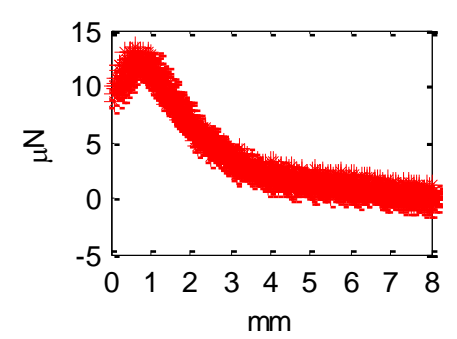

a) Time 1

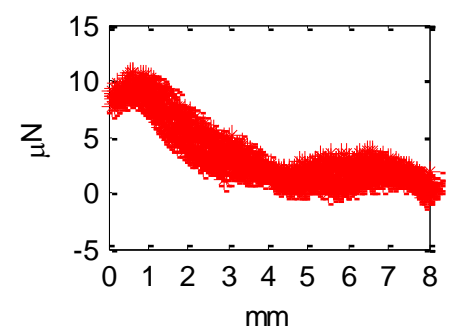

c) Time 3

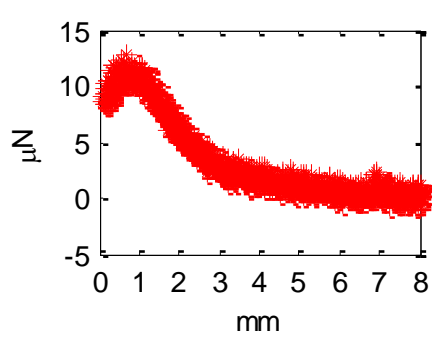

b) Time 2

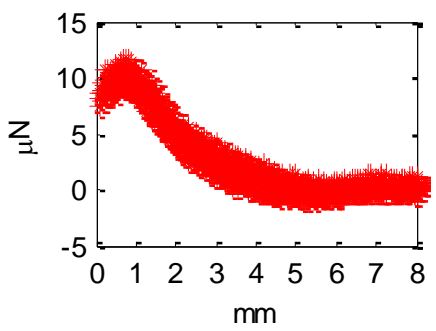

d) Time 4
Fig. 14 Evaluation of the repeatability of the magnetic force measurements. Four trials are made for a current of 0.6A. 
TABLE II Maximum measured force and corresponding distance for four different trials, input current $I=0.6 \mathrm{~A}$.

\begin{tabular}{ccc}
\hline Trial No. & $\begin{array}{c}\text { Maximum force } \\
(\mu \mathrm{N})\end{array}$ & $\begin{array}{c}\text { Corresponding } \\
\text { distance }(\mu \mathrm{m})\end{array}$ \\
\hline Time 1 & 13.56 & 599.0 \\
Time 2 & 12.86 & 670.5 \\
Time 3 & 12.40 & 879.0 \\
Time 4 & 11.69 & 799.2 \\
\hline Average value & 12.63 & 736.9 \\
Standard deviation & 0.79 & 125.8 \\
\hline
\end{tabular}

\section{CONCLUSION}

In this paper the magnetic field produced by an electromagnetic coil with an iron core is simulated with FEM analysis, and the magnetic field along the central axis is measured. The measurement result shows the effectiveness of the simulation. A three dimensional static magnetic analysis is performed to characterize the magnetic force applied to a nickel microrobot. The magnetic microforce along the central axis of the coil is also measured based on a developed experimental system. Comparison of the simulation and experimental results shows the effectiveness of the simulation of the force. The magnetic force applied to the microrobot can be up to $10 \mu \mathrm{N}$ which is sufficient to overcome adhesion and inertial forces. The simulator developed in this work will enable to control the position of the microrobot by controlling the current applied on each coil.

In future works the microrobot will be actuated by the magnetic field and its trajectory will be controlled based on the proposed simulator.

\section{ACKNOWLEDGMENT}

The authors thank the support of Project ECNANOMAN (European and Chinese Platform for Nano Handling, Assembly and Manufacturing). This work has also been supported by the Labex ACTION project (contract "ANR-11-LABX-01-01"), by the French RENATECH network and its FEMTO-ST technological facility, by the Equipex ROBOTEX project (contract "ANR-10-EQPX-44-01"), by the the French Agence
Nationale de la Recherche, through the LEMA project (contact "ANR 12 BS03 007 01") and by the Région Franche Comté.

\section{REFERENCES}

[1] B. J. Nelson, I. K. Kaliakatsos and J. J. Abbott, "Microrobots for Minimally Invasive Medicine,” Annu. Rev. Biomed. Eng., vol. 12, no. 1, pp. $55-85,2010$

[2] L. Feng, M. Hagiwara, A. Ichikawa, T. Kawahara and F. Arai, "Smooth enucleation of bovine oocyte by microrobot with local flow speed control in microchannel," Proc. IEEE/RSJ Int. Conf. Intell.Robots Syst., pp. 944-949, 2012.

[3] E. B. Steager, M. S. Sakar, C. Magee, M. Kennedy, A. Cowley and V. Kumar, "Automated biomanipulation of single cells using magnetic microrobots,” Int. J. Robot. Res., vol. 32, no. 3, pp. 346-359, 2013.

[4] H. Uvet, L. Feng, S. Ohashi, M. Hagiwara, T. Kawahara, Y. Yamanishi and F. Arai, "On-chip single particle loading and dispensing," Proc. IEEE Int. Conf. Robotics and Automation, pp. 3151-3156, 2011.

[5] S. Bouchebout, A. Bolopion, J.O. Abrahaminans, and S. Régnier, "An overview of multiple DoF magnetic actuated micro-robots," J. MicroNano Mech., pp. 97-113, 2012.

[6] S. Floyd, C. Pawashe, and M. Sitti, "Two-dimensional contact and noncontact micromanipulation in liquid using an untethered mobile magnetic microrobot," IEEE Trans. Robot., vol. 25, no. 6, pp. 1332 1342, 2009.

[7] M.P. Kummer, J.J. Abbott, B.E. Kratochvil, R. Borer, A. Sengul, and B.J. Nelson, "OcotMag: an electromagnetic system for 5-DoF wireless micromanipulation,” IEEE Trans. Robot., vol. 26, no. 6, pp. 1006-1017, 2010.

[8] J.D. Keuning, J.d. Vries, L. Abelmann and S. Misra, "Image-based magnetic control of paramagnetic microparticles in water, "Proc. IEEE/RSJ Int. Conf. Intell. Robots Syst., pp. 421-426, 2011.

[9] I.A. Ivan, G. Hwang, J. Agnus, M. Rakotondrabe, N. Chaillet, and S. Régnier, "First experiments on MagPieR: a planar wireless magnetic and piezoelectric microrobot," Proc. IEEE Int. Conf. Robotics and Automation, pp. 102-108, 2011.

[10] K.B. Yesin, K. Vollmers, and B.J. Nelson, "Modeling and control of untethered biomicrorobots in a fluidic environment using electromagnetic,” Int. J. Robot. Res., vol. 25, no. 5-6, pp. 527-536, 2006.

[11] Y.H. Ha, B.H. Han, and S.Y. Lee, "Magnetic propulsion of a magnetic device using three square-Helmholtz coils and a square-Maxwell coil," Med. Biol. Eng. Comput., vol. 48, pp. 139-145, 2010.

[12] M. Beleggia, M. De Graef, and Y. T. Millev, "The equivalent ellipsoid of a magnetized body," J. Phys. D: Appl. Phys. vol. 39, pp. 891-899, 2006. 